ARTÍCULO DE INVESTIGACIÓN

Revista Facultad de Salud - RFS - Julio - Diciembre de 2012;4-2:45-54

\title{
TOXICIDAD HEMATOLÓGICA ASOCIADA AL TRATAMIENTO CON SULFONAMIDAS Y PIRIMETAMINA EN PACIENTES VIH POSITIVOS $Y$ TOXOPLASMOSIS CEREBRAL EN UN HOSPITAL DE TERCER NIVEL EN COLOMBIA Hematologic toxicity associated with sulfonamides and pyrimethamine treatment in HIV positive patients with cerebral toxoplasmosis at tertiary-slevel hospital in Colombia
}

Carlos A. Gómez A. ${ }^{1}$, Sandra M. Gualtero ${ }^{1}$, Giovanna Botero J. ${ }^{2}$, Cindy L. Beltrán E. ${ }^{2}$, Francy L. Plazas ${ }^{2}$, Juan C. Cifuentes C. ${ }^{2}$, Miguel Sandoval C. ${ }^{2}$,

Dagoberto Santofimio ${ }^{3}$, Luis F. Durán ${ }^{4}$

Recibido: 13 de octubre de 2011 - Aceptado: 29 de noviembre de 2012

${ }^{1}$ Unidad de Infectología, Hospital Universitario Hernando Moncaleano Perdomo, Neiva-Colombia.

${ }^{2}$ Estudiantes de Medicina, Universidad Surcolombiana, Neiva-Colombia.

${ }^{3}$ Unidad de Epidemiología, Hospital Universitario Hernando Moncaleano Perdomo, Neiva-Colombia.

${ }^{4}$ Departamento de Medicina Interna, Universidad

Surcolombiana, Hospital Universitario Hernando Moncaleano Perdomo, Neiva-Colombia.

Correspondencia: giovibj@hotmail.com

\section{Resumen}

Objetivo: Determinar la frecuencia efectos adversos hematológicos asociados al tratamiento para toxoplasmosis cerebral en pacientes VIH/ SIDA. Diseño: Serie de casos retrospectiva.

Lugar: Hospital Universitario Hernando Moncaleano Perdomo (HMP), Neiva-Colombia.

Población: Pacientes con toxoplasmosis cerebral y VIH/SIDA atendidos en el servicio de Infectología entre 2006-2009.

Resultados: 51 pacientes fueron evaluados durante el periodo de estudio. 40 (78\%) fueron hombres. El promedio de edad fue 33 años. 25 pacientes presentaban toxoplasmosis cerebral como primer marcador de Infección VIH. El 60,7\% de los casos presentaron toxicidad hematológica. $42 \%$ de los pacientes presentaron anemia previa al tratamiento. El pico de aparición de toxicidad medular fue al sexto día de inicio del esquema de tratamiento. El esquema antitoxoplasma que más comúnmente fue asociado a mielotoxicidad fue la combinación de pirimetamina/sulfadoxina, trimetoprimsulfametoxazol y clindamicina en $48 \%$ de los casos.

Palabras clave: toxoplasmosis cerebral, VIH- SIDA, mielotoxicidad, exantema, pirimetamina-sulfadoxina, trimetoprimsulfametoxazol. 


\begin{abstract}
Objective: To determine the frequency of hematologic adverse effects associated with treatment for cerebral toxoplasmosis in HIV / AIDS patients. Design: Retrospective case series. Location: University Hospital Hernando Moncaleano Perdomo (HMP), Neiva, Colombia.

Population: Patients with cerebral toxoplasmosis and HIV / AIDS treated at the infection service unit between 2006 and 2009.

Población: Pacientes con toxoplasmosis cerebral y VIH/SIDA atendidos en el servicio de infectología entre 2006-2009.

Results: 51 patients were evaluated during the study period. 40 (78\%) were men. The average age was 33 years. 25 patients had cerebral toxoplasmosis as the first marker of HIV infection. $60.7 \%$ of cases had hematologic toxicity. $42 \%$ of patients had anemia before treatment. The peak onset of bone marrow toxicity was the sixth day of the start of treatment schedule. The anti-toxoplasma scheme that was most commonly associated with myelotoxicity was the combination of pyrimethamine/sulfadoxine, trimethoprim-sulfamethoxazole and clindamycin in $48 \%$ of cases.
\end{abstract}

Keywords: cerebral toxoplasmosis, HIV-AIDS, myelotoxicity, skin rash, pyrimethamine-sulfadoxine, trimethoprim-sulfamethoxazole.

\section{INTRODUCCIÓN}

La toxoplasmosis cerebral es la principal infección oportunista del sistema nervioso central en pacientes con VIH-SIDA. En Colombia hay pocos estudios; Silva y col.(1) describieron una serie de 21 episodios durante los primeros años de la epidemia en Bogotá (1988-1993). Corral y col.(2) describen 33 casos vistos entre 1988 y 1994 en Cali. En estados unidos ocurren aproximadamente 2000 casos de encefalitis causada por toxoplasmosis(2). En Cúcuta se reportaron al año 2005131 casos de infecciones oportunistas del Sistema nervioso central en pacientes VIH positivos en los últimos 10 años, describiendo que el $56 \%$ de estos pacientes presentaron toxoplasmosis cerebral(3). Al año 2006 se reportaron 21 casos de toxoplasmosis cerebral como infección oportunista $(62 \%)^{(4,5)}$.

En el Departamento del Huila según las estadísticas de la Secretaría de Salud existen registrados entre 1998 y la semana 24 del año 2008752 casos de personas infectadas con el virus del VIH entre la edad de 15 a 49 años de los cuales 511 se encuentran en el municipio de Neiva. Durante el periodo del estudio ingresaron a la unidad de infectología del Hospital Universitario Hernando Moncaleano Perdomo (HUHMP) un total de 209 pacientes VIH positivos, de los cuales 89 presentaron toxoplasmosis cerebral ${ }^{(6)}$.

La toxoplasmosis cerebral es una enfermedad cuyo agente etiológico es el protozoo Toxoplasma Gondii. La enfermedad parece ocurrir casi exclusivamente por la reactivación de un quiste tisular latente. En la era pre terapia HAART la incidencia a 12 meses en pacientes con inmunosupresión avanzada que fueron seropositivos para T. Gondii y no recibieron profilaxis anti- toxoplasma es de aproximadamente 33\%. El mayor riesgo de desarrollar la enfermedad es el conteo de CD4+ menor a $50 \mathrm{cel} / \mathrm{ul}$. La presentación clínica más común entre pacientes VIH positivos es una encefalitis focal que cursa con cefalea, confusión, déficit motor y fiebre. El examen físico puede mostrar anormalidades neurológicas focales, y en la ausencia de tratamiento la enfermedad puede progresar a convulsiones, estupor y coma. Formas como la Coriorretinitis, 
neumonía y evidencia de compromiso multisistémico pueden ser observados en la forma de infección diseminada pero son raros en esta población ${ }^{(7)}$.

El tratamiento de primera línea según el Centro para el control y la prevención de las enfermedades (CDC) se basa en la combinación de pirimetamina más sulfadiazina y leucovorin con nivel de evidencia $\mathrm{AI}^{(7)}$. En nuestro país no se dispone de pirimetamina ni sulfadiazina, por lo que se utiliza el medicamento disponible que es la combinación de pirimetamina- sulfadoxina (conocido como Falcidar). En caso se tenga alguna contraindicación de uso de alguno de estos medicamentos se utilizan otras alternativas con menos soporte científico de efectividad en el manejo de la toxoplasmosis como trimetropim sulfametoxazol, clindamicina, atovacuona. pirimetamina y las sulfonamidas ${ }^{(8,9)}$. Estos 2 últimos se han relacionado con la aparición de efectos adversos tales como trombocitopenia, leucopenia, anemia hemolítica y reacciones cutáneas; razón por la que se adiciona al tratamiento Ácido folínico 10 a 25 mg/día el cual está indicado tanto en la prevención de este efecto adverso como en el tratamiento de la complicación ${ }^{(7,10)}$.

En nuestro país no se cuenta con estudios que muestren la frecuencia de tales reacciones adversas en pacientes VIH positivos. El presente trabajo tiene como objetivo describir y definir el impacto de los efectos adversos relacionados con el tratamiento con pirimetamina y sulfonamidas en pacientes con SIDA tratados en el Hospital Universitario Hernando Moncaleano Perdomo durante el período de estudio que presentan como enfermedad oportunista toxoplasmosis cerebral según criterios radiológicos y clínicos de la CDC.

\section{MAteriales y MÉtodos}

Estudio de serie de casos retrospectivo a partir de pacientes hospitalizados en la Uni- dad de Infectología del Hospital Universitario Hernando Moncaleano Perdomo en Neiva. Los criterios de inclusión fueron pacientes diagnosticados con VIH/SIDA con western Blott positivo, hallazgos clínicos, radiológicos: tomográficos compatibles con lesión ocupando espacio en sistema nervioso central y serología ig $\mathrm{G}$ toxoplasmosis positiva y que iniciaran manejo médico con componentes sulfamidas, pirimetamina. Se excluyeron pacientes sin confirmación de su estado VIH y aquellos que se evidenciara otro agente etiológico que explicara las lesiones en el SNC.

A partir del servicio de archivo de la institución, se realizó una revisión sistemática de historias clínicas de pacientes que cumplieran criterios de inclusión. El periodo de estudio fue desde enero de 2006 hasta enero de 2009. Se elaboró un instrumento (ficha epidemiológica) para la recolección de la información. Este instrumento fue unificado y probado previamente en una prueba piloto para aclarar criterios entre todos los investigadores y garantizar la homogeneidad de la información recolectada. Para el análisis estadístico se utilizó el programa estadístico epiinfo versión 3.4 2007. Se mantuvo la confidencialidad de la historia clínica y del paciente. Nuestro estudio contó con la aprobación de la unidad de Infectología, el departamento de medicina Interna y la dirección científica de nuestra institución para la revisión y utilización de la información a partir de historias clínicas. No se presentaron conflictos de intereses, el proyecto fue financiado exclusivamente por los autores.

\section{RESUltados}

51 pacientes cumplieron los criterios de inclusión. 40 (78\%) fueron hombres. El promedio de edad observado en hombres y mujeres fue de 33,4 y 33,1 respectivamente. El 57\% provenían de la ciudad de Neiva. La mitad de los pacientes debutaban con toxoplasmosis cerebral como la primera infección oportunista. 43 
(84\%) de los pacientes tenían entre 20 y 40 años de edad y menos de 100 células $/ \mu \mathrm{L}$ de conteo de linfocitos (Tabla 1).

De los 31 pacientes que presentaron mielotoxicidad el 9,6\% padecían alguna comorbilidad, se documentan la farmacodependencia y la hepatitis viral, la neuropatía y la enfermedad renal se desencadenaron durante el curso de la enfermedad (Tabla 2).

Toxicidad asociada al tratamiento con sulfonamidas. El $60.7 \%$ de los casos presentaron toxicidad hematológica. El 41,9\% de los casos con mielotoxicidad presentaron anemia antes de iniciar el tratamiento antitoxoplasma. El hallazgo más frecuente de mielotoxicidad fue trombocitopenia (94\%), seguido de leucopenia $(64,5 \%)$, hemoglobina mayor de 7 $\mathrm{mg} / \mathrm{dl}$ y neutropenia (29\%) (Figura 1).

Esquemas de tratamiento. El tratamiento para Toxoplasmosis cerebral que más se relacionó con Mielotoxicidad consistió en la combinación pirimetamina sulfadoxina $75 / 1500 \mathrm{mg}$ vía oral DIA, clindamicina 2400 mg día, asociado a ácido folínico $15 \mathrm{mg}$ vía oral día y como

Tabla 1. Características demográficas y clínicas de pacientes VIH/SIDA con toxoplasmosis cerebral en HUHMP 2006-2009.

\begin{tabular}{lcccc}
\hline Variables & N (Porcentaje) & Mujeres & Hombres & Total \\
\hline Procedencia - Total & & & & $51(100 \%)$ \\
& Neiva & $4(40)$ & $25(60,9)$ & 29 \\
& Huila & $4(40)$ & $12(29,2)$ & 16 \\
& Otros & $2(20)$ & $4(9,6)$ & 6 \\
Debut de SIDA con & & & & \\
toxoplasmosis cerebral & & $5(50)$ & $20(49)$ & $25(50 \%)$ \\
Grupos de edad (años) & $20-40$ & $8(80)$ & $35(85,4)$ & 43 \\
& $41-60$ & $2(20)$ & $5(12,2)$ & 7 \\
& $>61$ & 0 & $1(2,4)$ & 1 \\
Conteo de linfocitos CD4+ al momento de & & & & \\
diagnóstico toxoplasmosis cerebral (Cel/mm mm $\left.^{3}\right)$ & $<100$ & $8(80)$ & $35(85,4)$ & $43(84 \%)$ \\
& $100-200$ & $2(20)$ & $6(14,6)$ & $8(16 \%)$ \\
\hline Total & & $\mathbf{1 0}(\mathbf{1 0 0})$ & $\mathbf{4 1 ( 1 0 0 )}$ & $\mathbf{5 1}$ \\
\hline
\end{tabular}

Tabla 2. Frecuencia de comorbilidades y mielotoxicidad.

\begin{tabular}{lcc}
\hline & $\begin{array}{c}\text { Mielotoxicidad } \\
\text { n (porcentaje) }\end{array}$ & $\begin{array}{c}\text { Desenlace } \\
\text { n (porcentaje) }\end{array}$ \\
\hline Farmacodependencia & $1(3,2)$ & salida \\
Hepatitis viral B & 0 & salida \\
Neuropatía periférica distal & $1(3,2)$ & muerte \\
Enfermedad renal crónica & $1(3,2)$ & muerte \\
\hline Total & $\mathbf{3 ( 9 , 6 )}$ & \\
\hline
\end{tabular}



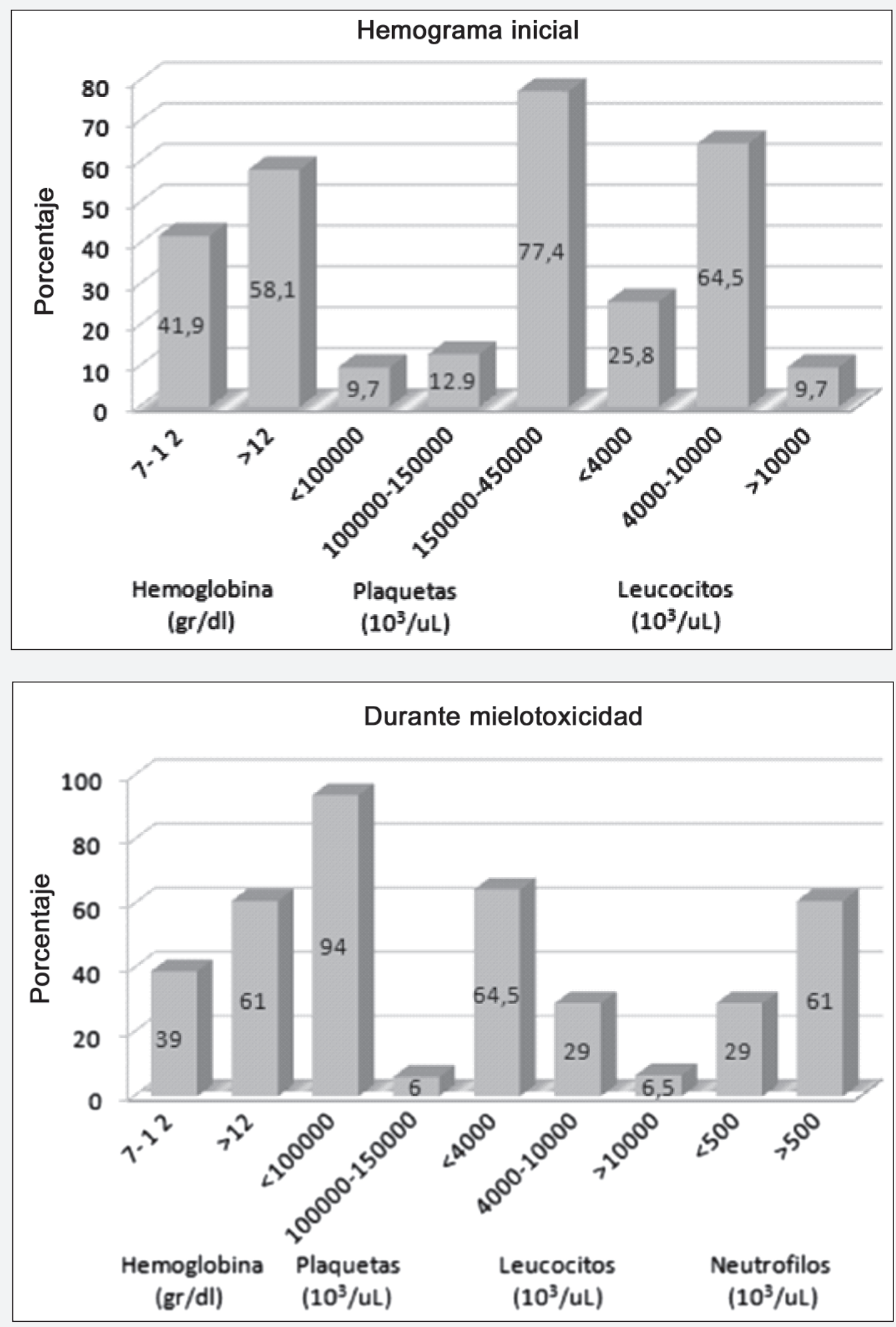

Figura 1. Hemograma inicial y durante los episodios de toxicidad hematológica de 31 pacientes con toxoplasmosis cerebral y VIH/SIDA. 
profilaxis para pneumocistis jiroveci recibieron trimetoprim sulfametoxazol 160/800 mg vía oral día (Tabla 3), con una aparición promedio del efecto adverso entre el quinto y décimo día, y una recuperación posterior en el mismo periodo de tiempo (Figura 2).

Dosis vs. toxicidad. La combinación de pirimetamina-sulfadoxina, más Tmp-smx más Clindamicina $(75 / 1500+160 / 800+2400)$ mg/día fue en el esquema de tratamiento con mayor frecuencia con mielotoxicidad (Figura 3).

Manejo de toxicidad. El manejo de la mielotoxicidad se basó en la suspensión de medicamentos en el 53\% de los pacientes y de estos el 58\% correspondió a la suspensión de pirimetamina/sulfadoxina, el 13\% TMP/SMX, $3 \%$ a la suspensión de Clindamicina; y además la disminución de la dosis en un $8 \%$ de los pacientes (Tabla 4).

\section{DISCUSIÓN}

El presente estudio describe las reacciones adversas presentadas en un grupo de 51 pacientes con infección VIH/SIDA que recibieron tratamiento de primera línea para toxoplasmosis cerebral y profilaxis para pneumocistis con

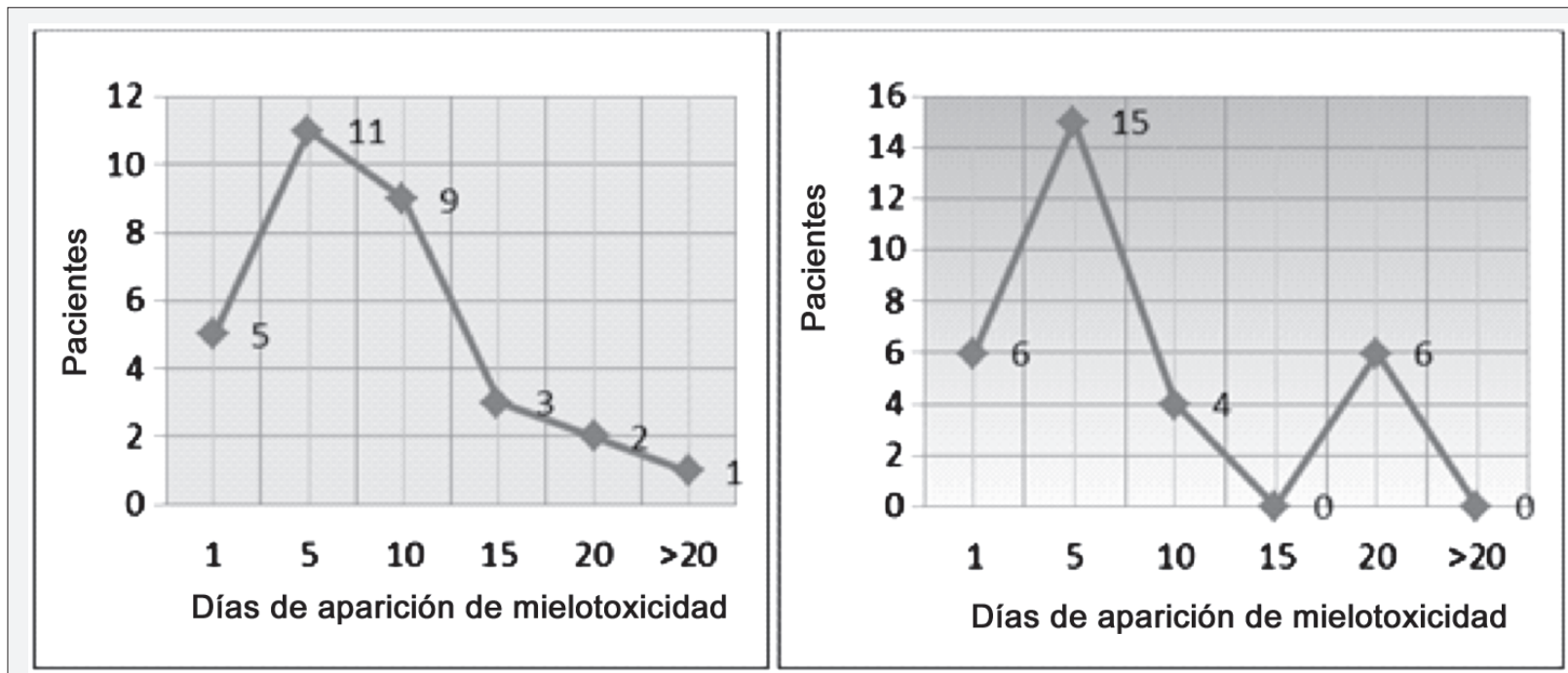

Figura 2. Distribución en el tiempo de 31 pacientes con toxicidad hematológica. Comparación del tiempo de aparición Vs. Tiempo de recuperación de la mielotoxicidad.

Tabla 3. Esquemas de tratamiento Anti-Toxoplasma y profilaxis contra Pneumocistis jiroveci durante la hospitalización de 31 pacientes con toxicidad hematológica.

Esquema de tratamiento
Toxicidad hematológica (Porcentaje)

Pirimetamina-Sulfadoxina

Pirimetamina-Sulfadoxina/TMP-SMX

Pirimetamina-Sulfadoxina / Clindamicina

TMP-SMX/Clindamicina

Pirimetamina-Sulfadoxina/TMP-SMX/Clindamicina

$\begin{array}{rr}1(3,2) & \\ 3(9,7) & \\ 10(32,3) & 17 \\ 2(6,4) & 2 \\ 15(48,4) & 22\end{array}$

Pacientes en tratamiento 


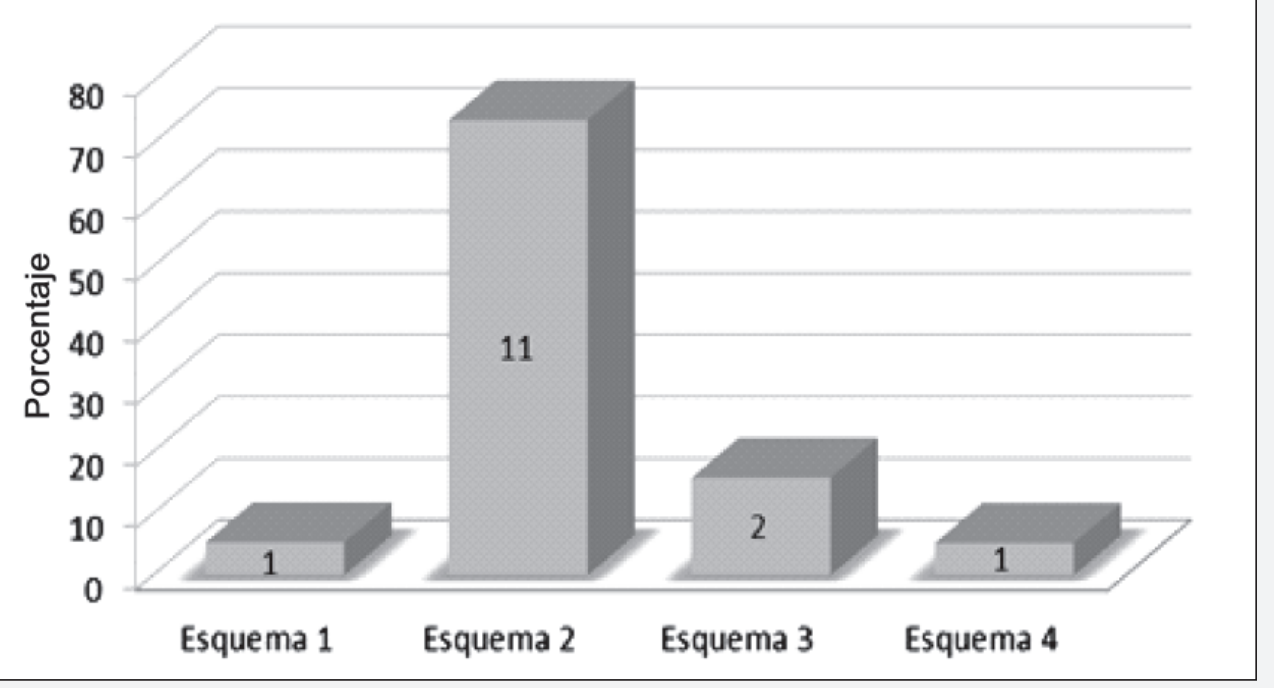

Figura 3. Esquema de dosificación del esquema Pirimetamina/Sulfadoxina /TMP-SMX y Clindamicina en 15 pacientes con toxicidad hematológica.

* Los rótulos indican el número de pacientes.

Pirimetamina-sulfadoxina/TMP-SMX/Clindamicina

Esquema 1 (25/500+160/800+1800) mg/día, Esquema 2 (75/1500+160/800+2400) mg/ día, Esquema 3 (50/1000+160/800+2400) mg/día, Esquema 4 (1100/2000+1280/ 6400+2400) mg/día.

Tabla 4. Manejo de la mielotoxicidad.

\section{Medicamentos}

Suspensión tto antitox

Pirimetmina/sulfadoxina $18(58,1)$

TMP-SMX

$4(12,9)$

$\begin{array}{lr}\text { Pirimetmina/sulfadoxina -TMP-SMX } & 4(12,9)\end{array}$

Clindamicina

Total

$27(100)$

\section{Disminución dosis}

Pirimetmina/sulfadoxina $\quad 1(25)$

$\begin{array}{lr}\text { TMP-SMX } & \text { 2(50) }\end{array}$

TMP-SMX - Clindamicina $\quad 1(25)$

Total

$\underline{4(100)}$

trimetoprim-sulfametoxazol (TMPSMX). La combinación pirimetamina sulfadiazina no se encuentra disponible en nuestro país. La disponibilidad de pirimetamina como monote- rapia rara vez es una opción posible, ante lo cual pirimetamina sulfadoxina (FALCIDAR) en combinación con clindamicina fue utilizada como tratamiento de primera línea, basados en 
el protocolo nacional de manejo de toxoplasmosis cerebral. Este esquema antitoxoplasma ha demostrado efectividad clínica en estudios realizados en nuestro medio, y ha sido descrito en estudios franceses que han utilizado el Falcidar para el tratamiento de toxoplasmosis congénita y perinatal ${ }^{(11)}$.

En cualquier tratamiento médico que involucre principios terapéuticos, existe el riesgo de desarrollar efectos adversos. Los efectos adversos más comunes asociados al uso de pirimetamina y sulfas son la hematotoxicidad y las reacciones dermatológicas tipo exantema urticaria y la anafilaxis. Estas reacciones se presentan incluso con el uso de dosis terapéuticas ${ }^{(12)}$. La frecuencia de la mielotoxicidad en pacientes VIH con toxoplasmosis tratados con esta combinación, en nuestro estudio fue el 60,9\%. Estos resultados concuerdan con lo descrito en la literatura. Leport y col. reportaron hematoxicidad relacionada con el uso de pirimetamina en $60 \%$ de 35 pacientes con infección VIH Sida en tratamiento de toxoplasmosis cerebral. Los efectos adversos de nuestro estudio fueron mayores en el grupo de terapia combinada. Esto puede ser explicado por la probabilidad de potenciar los efectos hematológicos con el uso de tmp-smx de manera rutinaria para la profilaxis antipneumocistis, cabe resaltar que la dosis del Falcidar no está estandarizada en nuestro hospital y se utilizan dosis superiores al protocolo colombiano de vihtoxoplasmosis, las cuales se relacionaron con mayor frecuencia a toxicidad hematológica; según la literatura la vida media de eliminación del Falcidar es de 170 horas pero la duración de la acción es de 24 horas lo que determina la dosis de carga e intervalo corto de administración cada 6 horas ${ }^{(8)}$.

Otro hallazgo referido en la literatura como factor relacionado con mayor frecuencia de efectos adversos cutáneos es el recuento de CD4, especialmente aquellos que presentan menos de 100 células de CD4, la prevalencia de recuentos de CD4+ menores a $100 \mathrm{cel} / \mathrm{dl}$ fue de $84 \%$ en nuestro estudio, lo cual está relacionado con lo reportado en estudios anteriores ${ }^{(13)}$.

En presencia de Hematotoxicidad, la línea celular más susceptible es la plaquetaria, en nuestra serie de casos la trombocitopenia se presentó en el 93\%. También se presentaron 9 casos de neutropenia en un periodo de 4 años siendo pocos, reporte también realizado por Van der Klauw en un período de 20 años descrito con una frecuencia mayor a 206 casos.

El tiempo promedio de aparición de mielotoxicidad en nuestra serie fue entre el sexto y el décimo día después de iniciar el tratamiento con un esquema pirimetaminasulfadoxina/tmp-smx/Clindamicina que concuerda con La revisión descrita por Richard H. Aster et al. En donde describen el curso de la trombocitopenia inducida por medicamentos. La aseveración de que las reacciones hematológicas tienen este curso típico, consistente en un paciente que se ha sensibilizado con un medicamento por cerca de una semana o de forma intermitente durante un período de tiempo más largo antes de la presentación de trombocitopenia con petequias; y una vez se suspende el medicamento causante del proceso, la recuperación hematológica se da en un periodo de tiempo equitativo a la aparición ${ }^{(14)}$.

El manejo más frecuentemente instaurado cuando se presentaron casos de mielotoxicidad y exantema fue la suspensión de medicamentos sospechosos; Pirimetamina/Sulfadiaxina fue la combinación farmacéutica más frecuentemente suspendida en $58,1 \%$ de los casos, de conformidad con lo reportado en la literatura. Otras medidas de soporte como la transfusión de plaquetas pueden ser consideradas para el manejo de trombocitopenias severas con riesgo de sangrado mayor. El uso de Globulina Inmune intravenosa ${ }^{(15)}$ y la plasmaféresis no han demostrado efectividad clínica en el manejo de trombocitopenias severas asociadas al uso de sulfas ${ }^{(16)}$. 
Una vez establecido, probablemente persista la sensibilidad de drogas indefinidamente. Por lo tanto, los pacientes deben ser aconsejados para evitar permanentemente la medicación que se cree es la causa de la trombocitopenia. Afortunadamente, los anticuerpos inducidos por las drogas tienden a ser específicas para la droga sensibilizante ${ }^{(17)}$ y los pacientes suelen tolerar fármacos equivalentes, incluso aquellos con estructuras muy similares.

La presencia de efectos adversos genera un impacto importante tanto en el tiempo de duración del tratamiento de la enfermedad oportunista como la estancia hospitalaria y la aparición de otras complicaciones secundarias a sangrado, infecciones nosocomiales. Los resultados de nuestro estudio permitieron concertar un cambio en el esquema actual anti toxoplasma de nuestra institución. Como resultado del aumento de reacciones hematológicas cuando dos componentes sulfas eran utilizados, se decidió iniciar un estudio piloto que evaluara la efectividad de con uso de pirimetamina monoterapia sin sulfadoxina, en combinación con clindamicina. La dosis de TMPSMX como profilaxis antipneumocistis se estableció a 80/400 mg vía oral tres veces por semana. El uso generalizado de ácido folínico a dosis mayores que las recomendadas en la literatura. La eficacia clínica y la evaluación de efectos adversos con este nuevo esquema de dosis es esperado para realizar una comparación fiable entre nuestra propia población de pacientes VIH.

\section{Agradecimientos}

Los autores expresan sus agradecimientos al Hospital Universitario de Neiva, al Programa de Medicina de la Universidad Surcolombiana y al Comité Editorial de la Revista Facultad de Salud por su apoyo y colaboración en el desarrollo de este artículo de investigación.

\section{REFERENCIAS}

1. Fauci B, Hauser L, Loscalzo J. Harrison principios de medicina interna. XVII edición; Mc Graw Hill 2009;7(282):1181-1184.

2. Silva F, Torres A, Prada G. Encefalitis por toxoplasma y SIDA: Análisis de 27 episodios. Revista Panamericana de Infectología 1997;1:4-9.

3. Lizarazo J, Castro F, M. de Arco. Infecciones oportunistas del sistema nervioso central en pacientes con VIH atendidos en el Hospital Universitario Erasmo Meoz, Cúcuta, 1995 2005, Infectio, 10 2006;226-231.

4. Osorio JC, Vallejo GS, Franco D. Determinación de las características clínico-epidemiológicas de la neuroinfección en pacientes con diagnóstico de VIH/sida en el departamento del Quindío. Infectio 2007;173-182.

5. Cardona N, Basto N, Gomez JE. detection of Toxoplasma DNA in the Peripheral Blood of HIVPositive Patients with Neuro-opportunistic Infections by a Real-Time PCR Assay. Journal of Neuroparasitology 2011.

6. Secretaría de salud departamental, Sistema de Vigilancia epidemiológica (SIVIGILA), Boletín epidemiológico del Huila, 2008.

7. Centers for Disease Control and Prevention. Guidelines for Prevention and Treatment of Opportunistic Infections in HIV-Infected Adults and Adolescents Recommendations from CDC, the National Institutes of Health, and the HIV Medicine Association of the Infectious Diseases Society of America 2009;58:12-13.

8. Corral RH, Varela A, González I, Gutiérrez MI, et al. Toxoplasmosis en pacientes con infección por VIH. Boletín SIEI (ISS Cali) 1996;2:11-16.

9. Gómez JE, Alvarado F, Hernández C, et al. Tratamiento de la fase aguda de la toxoplasmosis cerebral con Clindamicina - Falcidar (pirimetamina - sulfadoxina) en pacientes infectados por VIH. Infectio 2001;5(3):162-168.

10. Fauci B, Hauser L, Loscalzo J. Harrison principios de medicina interna. XVII edición; Mc Graw Hill 2009;1199.

11. Gómez JE, Alvarado F, Hernández C, et al. Tratamiento de la fase aguda de la toxoplasmosis cerebral con Clindamicina - Falcidar (pirimetamina - sulfadoxina) en pacientes infectados por VIH. Infectio 2001;5(3):162-168.

12. Erik R, Vandendries, Reed E. Drews. DrugAssociated Disease: Hematologic Dysfunction. Crit Care Clin 2006;22:347-355. 
13. Van der Ven AJ, Koopmans PP, van der Meer JW. Adverse reactions to co-trimoxazole in HIV infection. Lancet 1991;338(8764):431-433.

14. Richard H. Aster, and Daniel W. Bougie. DrugInduced Immune Thrombocytopenia. N Engl J Med 2007;357:580-587.

15. Ray JB, Brereton WF, Nullet FR. Intravenous immune globulin for the treatment of presumed quinidine-induced thrombocytopenia. DICP 1990;24:693-695.
16. Pourrat $\mathrm{O}$. Treatment of drug-related diseases by plasma exchanges. Ann Med Interne (París) 1994;145:357-360.

17. Christie DJ, Weber RW, Mullen PC, Cook JM, Aster RH. Structural features of the quinidine and quinine molecules necessary for binding of drug-induced antibodies to human platelets. J Lab Clin Med 1984;104:730-740. 